\title{
Final-boundary value problem in the non-classical treatment for a sixth order pseudoparabolic Equation
}

\author{
Ilgar Gurbat oglu Mamedov
}

Institute of Cybernetics Azerbaijan National Academy of Sciences, B. Vahabzade St.9, Baku city, AZ 1141, Azerbaijan Republic

Email address:

ilgar-mammadov@rambler.ru

\section{To cite this article:}

Ilgar Gurbat oglu Mamedov. Final-Boundary Value Problem in the Non-Classical Treatment for a Sixth Order Pseudoparabolic Equation. Applied and Computational Mathematics. Vol. 2, No. 3, 2013, pp. 96-99. doi: 10.11648/j.acm.20130203.15

\begin{abstract}
In this paper substantiated for a differential equation of pseudoparabolic type with discontinuous coefficients a final-boundary problem with non-classical boundary conditions is considered, which requires no matching conditions. The considered equation as a pseudoparabolic equation generalizes not only classic equations of mathematical physics (heatconductivity equations, string vibration equation) and also many models differential equations (telegraph equation, Aller's equation , moisture transfer generalized equation, Manjeron equation, Boussinesq-Love equation and etc.). It is grounded that the final-boundary conditions in the classic and non-classic treatment are equivalent to each other, and such boundary conditions are demonstrated in geometric form. Even from geometric interpretation can see that the grounded non-classic treatment doesn't require any additional conditions of agreement type. Thus, namely in this paper, the non-classic problem with final-boundary conditions is grounded for a pseudoparabolic equation of sixth order. For simplicity, this was demonstrated for one model case in one of S.L. Sobolev anisotropic space $W_{p}^{(4,2)}(G)$.
\end{abstract}

Keywords: Final-Boundary Value Problem, Pseudoparabolic Equations, Equations with Discontinuous Coefficients

\section{Introduction}

Urgency of investigations conducted in this field is explained by appearance of local and non-local problems for discontinuous coefficients equations connected with different applied problems. Such type problems arise for example, while studying the problems of moisture, transfer in soils, heat transfer in heterogeneous media, diffusion of thermal neutrons in inhibitors, simulation of different biological processes, phenomena and etc.

Pseudoparabolic equations are attracted for sufficiently adequate description of a great deal of real processes occurring in the nature, engineering and etc. In particular, many processes arising in the theory of fluid filtration in cracked media are described by discontinuous coefficient pseudoparabolic equations .

In the present paper, here consider final-boundary problem for sixth order equation with discontinuous coefficients. The coefficients in this pseudoparabolic equation are not necessarily differentiable; therefore, there does not exist a formally adjoint differential equation making a certain sense. For this reason, this question cannot be investigated by the well-known methods using classical integration by parts and Riemann functions or classical-type fundamental solutions. The theme of the present paper, devoted to the investigation final-boundary problem for sixth order differential equations of pseudoparabolic type, according to the above-stated is very actual for the solution of theoretical and practical problems. From this point of view, the paper is devoted to the actual problems of mathematical physics and computational mathematics.

\section{Problem Statement}

Consider the equation

$$
\begin{gathered}
\left(V_{4,2} u\right)(x, y) \equiv D_{x}^{4} D_{y}^{2} u(x, y)+a_{3,2}(x, y) D_{x}^{3} D_{y}^{2} u(x, y)+ \\
+a_{4,1}(x, y) D_{x}^{4} D_{y} u(x, y)+\sum_{i=0}^{4} \sum_{\substack{j=0 \\
i+j<5}}^{2} a_{i, j}(x, y) D_{x}^{i} D_{y}^{j} u(x, y)=Z_{4,2}(x, y) \in L_{p}(G) .
\end{gathered}
$$


Here $u(x, y)$ is a desired function determined on $G$; $a_{i, j}(x, y)$ are the given measurable functions on $G=G_{1} \times G_{2}$, where $G_{k}=\left(0, h_{k}\right), k=1,2 ; Z_{4,2}(x, y)$ is a given measurable function on $G ; D_{t}^{n}=\partial^{n} / \partial t^{n}$-is a generalized differentiation operator in S.L.Sobolev sense and $D_{t}^{0}$-is an identity transformation operator.

Equation (1) is a hyperbolic equation possessing two real characteristics $x=$ const, $y=$ const , the first of which is four-fold, the second is two-fold. Therefore, in some sense, we can consider equation (1) as a pseudoparabolic equation [1]. This equation is a Boussinesq - Love generalized equation from the vibrations theory [2] and Aller's equation under mathematical modeling [3, p.261] of the moisture absorption process in biology.

In the present paper we consider equation (1) in the general sense when the coefficients $a_{i, j}(x, y)$ are non smooth functions satisfying only the following conditions:

$$
\begin{gathered}
a_{i, j}(x, y) \in L_{p}(G), i=\overline{0,3} j=\overline{0,1} \\
a_{4, j}(x, y) \in L_{\infty, p}^{x, y}(G), j=\overline{0,1} \\
a_{i, 2}(x, y) \in L_{p, \infty}^{x, y}(G), i=\overline{0,3 .}
\end{gathered}
$$

Under these conditions the solution $u(x, y)$ of equation (1) will be sought in S.L.Sobolev space

$$
W_{p}^{(4,2)}(G) \equiv\left\{u(x, y): D_{x}^{i} D_{y}^{j} u(x, y) \in L_{p}(G), i=\overline{0,4}, j=\overline{0,2}\right\}
$$

where $1 \leq p \leq \infty$. For equation (1) we can give the final boundary conditions of classic form as (see. Fig.1.)

$$
\left\{\begin{array}{c}
\left.u(x, y)\right|_{x=h_{1}}=\phi_{1}(y) ;\left.u(x, y)\right|_{y=h_{2}}=\psi_{1}(x) ; \\
\left.\frac{\partial u(x, y)}{\partial x}\right|_{x=h_{1}}=\phi_{2}(y) ;\left.\frac{\partial u(x, y)}{\partial y}\right|_{y=h_{2}}=\psi_{2}(x) ; \\
\left.\frac{\partial^{2} u(x, y)}{\partial x^{2}}\right|_{x=h_{1}}=\phi_{3}(y) ;\left.\frac{\partial^{3} u(x, y)}{\partial x^{3}}\right|_{x=h_{1}}=\phi_{4}(y) ;
\end{array}\right.
$$

where $\phi_{k}(y), k=\overline{1,4} \quad$ и $\psi_{1}(x), \psi_{2}(x)$ are the given measurable functions on $G$. Obviously, in the case of conditions (2), in addition to the conditions

$\phi_{k}(y) \in W_{p}^{(2)}\left(G_{2}\right), k=\overline{1,4} ; \quad \psi_{1}(x) \in W_{p}^{(4)}\left(G_{1}\right), \psi_{2}(x) \in W_{p}^{(4)}\left(G_{1}\right)$;

the given functions should satisfy also the following agreement conditions:

$$
\begin{cases}\psi_{1}\left(h_{1}\right)=\phi_{1}\left(h_{2}\right) ; & \psi_{2}\left(h_{1}\right)=\phi_{1}^{\prime}\left(h_{2}\right) ; \\ \psi_{1}^{\prime}\left(h_{1}\right)=\phi_{2}\left(h_{2}\right) ; & \psi_{2}^{\prime}\left(h_{1}\right)=\phi_{2}^{\prime}\left(h_{2}\right) ; \\ \psi_{1}^{\prime \prime}\left(h_{1}\right)=\phi_{3}\left(h_{2}\right) ; & \psi_{2}^{\prime \prime}\left(h_{1}\right)=\phi_{3}^{\prime}\left(h_{2}\right) ; \\ \psi_{1}^{\prime \prime \prime}\left(h_{1}\right)=\phi_{4}\left(h_{2}\right) ; & \psi_{2}^{\prime \prime \prime}\left(h_{1}\right)=\phi_{4}^{\prime}\left(h_{2}\right) .\end{cases}
$$

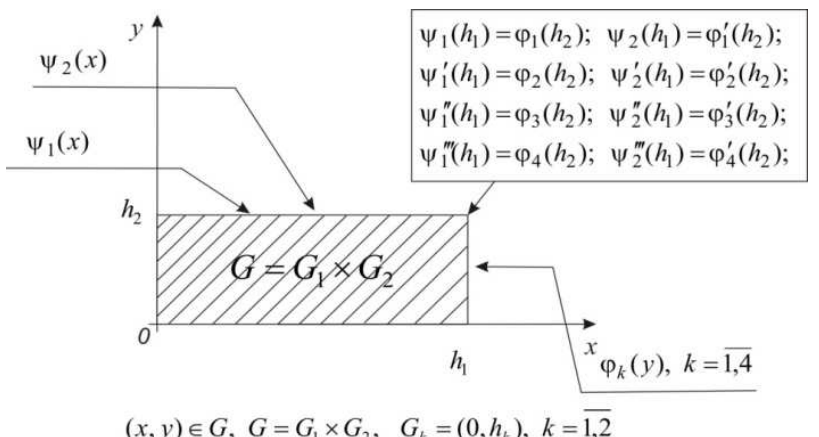

Fig 1. Geometrical interpretation of classic final-boundary conditions

Consider the following non - classical boundary conditions:

$\left\{\begin{array}{l}V_{i, j} u \equiv D_{x}^{i} D_{y}^{j} u\left(h_{1}, h_{2}\right)=Z_{i, j} \in R, i=\overline{0,3}, j=\overline{0,1} \\ \left(V_{4, j} u\right)(x) \equiv D_{x}^{4} D_{y}^{j} u\left(x, h_{2}\right)=Z_{4, j}(x) \in L_{p}\left(G_{1}\right), j=\overline{0,1} \\ \left(V_{i, 2} u\right)(y) \equiv D_{x}^{i} D_{y}^{2} u\left(h_{1}, y\right)=Z_{i, 2}(y) \in L_{p}\left(G_{2}\right), i=\overline{0,3}\end{array}\right.$

\section{Methodology}

There with, the important principal moment is that the considered equation possesses discontinuous coefficients satisfying only some $p$-integrability and boundedness conditions i.e. the considered pseudoparabolic operator has no traditional conjugated operator. In other words, the Riemann function for this equation can't be investigated by the classical method of characteristics. In the papers [4-5] The Riemann function is determined as the solution of an integral equation. This is more natural than the classical way for deriving the Riemann function. The matter is that in the classic variant, for determining the Riemann function, the rigid smooth conditions on the coefficients of the equation are required.

The Riemann's method does not work for differential equations with discontinuous coefficients.

In the present paper, a method that essentially uses modern methods of the theory of functions and functional analysis is worked out for investigations of such problems. In the main, this method it requested in conformity to pseudoparabolic equations of sixth order. Notice that, in this paper the considered equation is a generation of many model equations of some processes (for example, heatconductivity equations, telegraph equation, Aller's equation, moisture transfer generalized equation, Manjeron equation, equation, string vibrations equations and etc).

If the function $u \in W_{p}^{(4,2)}(G)$ is a solution of classic type 
final boundary value problem (1), (2), then it is also a determined by the following equalities: solution of problem (1), (4) for $Z_{i, j}, i=\overline{0,4}, j=\overline{0,2}$,

$$
\begin{gathered}
Z_{0,0}=\phi_{1}\left(h_{2}\right)=\psi_{1}\left(h_{1}\right) ; Z_{0,1}=\phi_{1}^{\prime}\left(h_{2}\right)=\psi_{2}\left(h_{1}\right) ; Z_{1,0}=\phi_{2}\left(h_{2}\right)=\psi_{1}^{\prime}\left(h_{1}\right) ; Z_{1,1}=\phi_{2}^{\prime}\left(h_{2}\right)=\psi_{2}^{\prime}\left(h_{1}\right) ; Z_{2,0}=\phi_{3}\left(h_{2}\right)=\psi_{1}^{\prime \prime}\left(h_{1}\right) ; \\
Z_{2,1}=\phi_{3}^{\prime}\left(h_{2}\right)=\psi_{2}^{\prime \prime}\left(h_{1}\right) ; Z_{3,0}=\phi_{4}\left(h_{2}\right)=\psi_{1}^{\prime \prime \prime}\left(h_{1}\right) ; Z_{3,1}=\phi_{4}^{\prime}\left(h_{2}\right)=\psi_{2}^{\prime \prime \prime}\left(h_{1}\right) ; Z_{4,0}(x)=\psi_{1}^{(I V)}(x) ; Z_{4,1}(x)=\psi_{2}^{(I V)}(x) ; \\
Z_{0,2}(y)=\phi_{1}^{\prime \prime}(y) ; Z_{1,2}(y)=\phi_{2}^{\prime \prime}(y) Z_{2,2}(y)=\phi_{3}^{\prime \prime}(y) ; Z_{3,2}(y)=\phi_{4}^{\prime \prime}(y) .
\end{gathered}
$$

It is easy to prove that the inverse one is also true. In other words, if the function $u \in W_{p}^{(4,2)}(G)$ is a solution of problem (1), (4), then it is also a solution of problem (1), (2) for the following functions:

$$
\begin{gathered}
\phi_{1}(y)=Z_{0,0}+\left(y-h_{2}\right) Z_{0,1}+\int_{h_{2}}^{y}(y-\tau) Z_{0,2}(\tau) d \tau ; \\
\phi_{2}(y)=Z_{1,0}+\left(y-h_{2}\right) Z_{1,1}+\int_{h_{2}}^{y}(y-\xi) Z_{1,2}(\xi) d \xi ; \\
\phi_{3}(y)=Z_{2,0}+\left(y-h_{2}\right) Z_{2,1}+\int_{h_{2}}^{y}(y-\eta) Z_{2,2}(\eta) d \eta ; \\
\phi_{4}(y)=Z_{3,0}+\left(y-h_{2}\right) Z_{3,1}+\int_{h_{2}}^{y}(y-v) Z_{3,2}(v) d v ; \\
\psi_{1}(x)=Z_{0,0}+\left(x-h_{1}\right) Z_{1,0}+\frac{\left(x-h_{1}\right)^{2}}{2 !} Z_{2,0}= \\
+\frac{\left(x-h_{1}\right)^{3}}{3 !} Z_{3,0}+\int_{h_{1}}^{x} \frac{(x-\mu)^{3}}{3 !} Z_{4,0}(\mu) d \mu ; \\
\psi_{2}(x)=Z_{0,1}+\left(x-h_{1}\right) Z_{1,1}+\frac{\left(x-h_{1}\right)^{2}}{2 !} Z_{2,1}+ \\
+\frac{\left(x-h_{1}\right)^{2}}{3 !} Z_{3,1}+\int_{h_{1}}^{x} \frac{(x-\rho)^{3}}{3 !} Z_{4,1}(\rho) d \rho ;
\end{gathered}
$$

Note has the functions (5)-(10) possess one important property, more exactly, agreement conditions (3) for all $Z_{i, j}$, having the above-mentioned properties are fulfilled for them automatically. Therefore, we can consider equalities (5) - (10) as a general form of all the functions $\phi_{k}(y), k=\overline{1,4}, \psi_{1}(x), \psi_{2}(x) \quad, \quad$ satisfying agreement conditions (3).

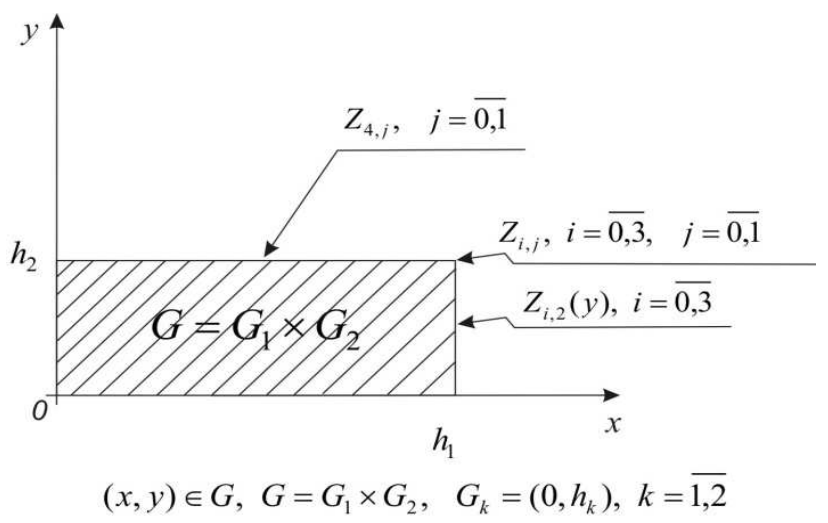

Fig 2. Geometric interpretation of final - boundary conditions in non-classical treatment.

\section{Result}

So, the classic form final-boundary problem (1), (2) and in non-classical treatment (1), (4) (see fig.2) are equivalent in the general case. However, the final-boundary value problem in non-classical treatment (1), (4) is more natural by the statement than problem (1), (2). This is connected with the fact that in the statement of problem (1), (4) the right sides of boundary conditions don't require additional conditions of agreement type. Note that such boundary value problems in non-classical treatment were considered in the author's papers [6-9].

\section{Discussion and Conclusions}

In the paper a non-classical type final-boundary problem is substantiated for a pseudoparabolic equation with nonsmooth coefficients and with a sixth order dominating derivative. Classic final-boundary conditions are reduced to non-classic final-boundary conditions by means of integral representations. Such statement of the final-boundary problem has several advantages:

1) No additional agreement conditions are required in this statement;

2) One can consider this statement as a final-boundary problem formulated in terms of traces in the S.L. Sobolev anisotropic space $W_{p}^{(4,2)}(G)$;

3) In this statement the considered equation is a generalization of many model equations of some processes (e.g. heat-conductivity equations , telegraph equation, Aller's equation, moisture transfer generalized equation, Manjeron equation, 
Boussinesq-Love equation, string vibrations equations and etc.).

\section{References}

[1] D.Colton, "Pseudoparabolic equations in one space variable", J. Different. equations, 1972, vol.12, No3, pp.559-565.

[2] A.P.Soldatov, M.Kh.Shkhanukov, "Boundary value problems with A.A.Samarsky general nonlocal condition for higher order pseudoparabolic equations", Dokl. AN SSSR, 1987, vol.297, No 3. pp.547-552 .

[3] A.M.Nakhushev, Equations of mathematical biology. M.: Visshaya Shkola, 1995, 301p.

[4] S.S.Akhiev, "Fundamental solution to some local and non local boundary value problems and their representations ", DAN SSSR, 1983, vol.271, No 2, pp.265-269.

[5] V.I.Zhegalov, E.A.Utkina, "On a third order pseudoparabolic equation”, Izv. Vuzov, Matem., 1999, No
10, pp.73-76.

[6] I.G.Mamedov, " A fundamental solution to the Cauchy problem for a fourth- order pseudoparabolic equation",Computational Mathematics and Mathematical Physics, 2009, volume 49, Issue 1, pp 93-104.

[7] I.G.Mamedov, "A non-classical formula for integration by parts related to Goursat problem for a pseudoparabolic equation”, Vladikavkazsky Matematicheskiy Zhurnal ,2011, vol.13, No 4, pp.40-51.

[8] I.G.Mamedov, "Goursat non - classic three dimensional problem for a hyperbolic equation with discontinuous coefficients", Vestnik Samarskogo Gosudarstvennogo Tekhnicheskogo Universiteta, 2010, No 1 (20), pp. 209-213.

[9] I.G.Mamedov, "Final-boundary value problem for a hyperbolic equation with multi triple characteristics", Functional analysis and its applications, Proc. of the International Conference devoted to the centenary of acad. Z.I.Khalilov,Baku,2011,pp.232-234. 